\title{
Assessing atherosclerotic plaque morphology: comparison of optical coherence tomography and high frequency intravascular ultrasound
}

\author{
M E Brezinski, G J Tearney, N J Weissman, S A Boppart, B E Bouma, M R Hee,
} A E Weyman, E A Swanson, J F Southern, J G Fujimoto

\begin{abstract}
Background-OCT can image plaque microstructure at a level of resolution not previously demonstrated with other imaging techniques because it uses infrared light rather than acoustic waves.

Objectives-To compare optical coherence tomography (OCT) and intravascular ultrasound (IVUS) imaging of in vitro atherosclerotic plaques.

Methods-Segments of abdominal aorta were obtained immediately before postmortem examination. Images of 20 sites from five patients were acquired with OCT (operating at an optical wavelength of $1300 \mathrm{~nm}$ which was delivered to the sample through an optical fibre) and a $30 \mathrm{MHz}$ ultrasonic transducer. After imaging, the microstructure of the tissue was assessed by routine histological processing.

Results-OCT yielded superior structural information in all plaques examined. The mean (SEM) axial resolution of OCT and IVUS imaging was 16 (1) and 110 (7), respectively, as determined by the point spread function from a mirror. Furthermore, the dynamic range of OCT was $109 \mathrm{~dB}$ compared with $43 \mathrm{~dB}$ for IVUS imaging.

Conclusions-OCT represents a promising new technology for intracoronary imaging because of its high resolution, broad dynamic range, and ability to be delivered through intravascular catheters.
\end{abstract}

Massachusetts General Hospital and Harvard Medical School,

Boston,

Massachusetts, USA:

The Cardiac Unit

ME Brezinski

N J Wiessman

A E Weyman

Pathology Department J F Southern

Department of Electrical Engineering and Computer

Science, The Research

Laboratory of

Electronics,

Massachusetts

Institute of

Technology,

Cambridge,

Massachusetts, USA

G J Tearney

$S$ A Boppart

B E Bouma

M R Hee

J G Fujimoto

Lincoln Laboratory,

Massachusetts

Institute of

Technology,

Lexington,

Massachusetts, USA

E A Swanson

Correspondence to:

Dr M E Brezinski, Bldg

Institute of Technology,

Cambridge, MA 02139,

Accepted for publication 15 October 1996

Keywords: atherosclerosis; plaque morphology; infrared light; intravascular ultrasound; optical coherence tomography
36-357, Massachusetts

(Heart 1996;77:397-403)

Sample

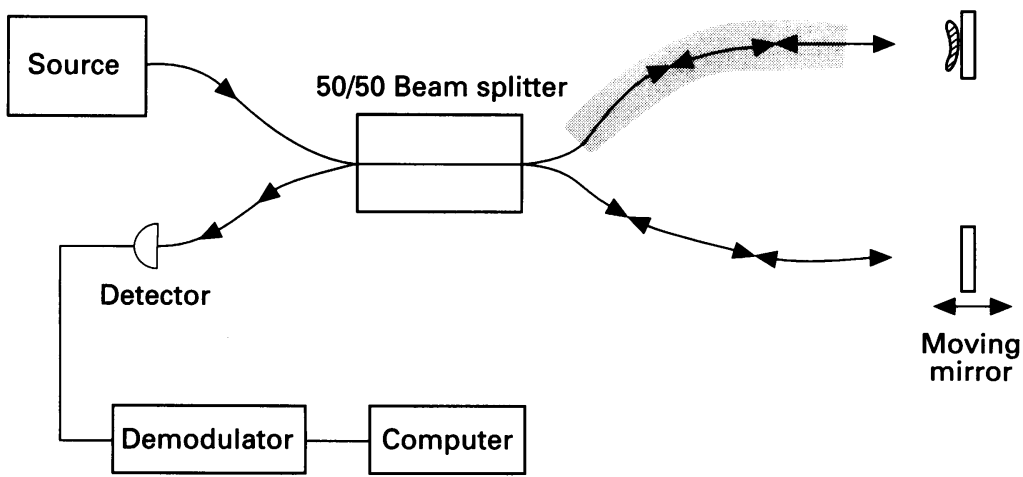

Figure 1 Schematic diagram of the OCT system. The shaded area represents the portion that would be present in the catheter.
The need for more detailed assessment of coronary microstructure, to improve risk stratification and guide interventional procedures, has driven the interest in developing new methods of imaging. Recently, high frequency intravascular ultrasound (IVUS) imaging has been used for the in vivo analysis of plaque morphology. ${ }^{1-3}$ IVUS imaging $(20-30 \mathrm{MHz})$ is superior to conventional angiography in its ability to guide stent deployment, diagnose dissection, assess the effectiveness of coronary intervention, and determine the extent of vessel obstruction. ${ }^{4-6}$ Clinical trials have been conducted to determine the impact of IVUS imaging on patient management. ${ }^{7}$ Analysis of plaque microstructure is limited with IVUS imaging, however, because of the relatively low resolution $(100-150 \mu \mathrm{m})$ and dynamic range (less than $50 \mathrm{~dB}$ ) ${ }^{8-10}$ The identification of plaque features predisposed to rupture and progression to acute coronary syndromes in particular has had limited success. Technologies with superior performance may be required for the in vivo diagnosis of plaque instability.

Optical coherence tomography (OCT) is a recently developed imaging technology that shows considerable promise as a method for high resolution intravascular imaging. ${ }^{11}$ OCT uses infrared light to produce tomographic images on a micrometre scale. OCT imaging is analogous to ultrasound B mode imaging, except that it performs imaging by measuring the intensity of reflected or back scattered light rather than acoustic waves. Tomographic images are produced in a manner similar to that of radar. An optical beam is scanned across the tissue and the reflected or back scattered light is measured as a function of range (depth) and transverse position. The result is a two or three dimensional dataset that represents the intensity of the optical back scattering in a section through the tissue. The intensity of the reflected light is displayed as a false colour or grey scale image.

Although penetration of OCT imaging in non-transparent tissues is limited to a few millimetres, the typical image resolution of OCT is $10-15 \mu \mathrm{m}$, almost 10 times greater than ultrasound assessment, magnetic resonance imaging, or computed tomography. OCT imaging with resolution as high as $4 \mu \mathrm{m}$ has been demonstrated in state of the art systems. ${ }^{12}$ Furthermore, OCT uses an optical fibre based design that can be readily integrated into catheters and endoscopes. OCT has been investigated, developed, and reduced to clinical practice for imaging transparent tis- 

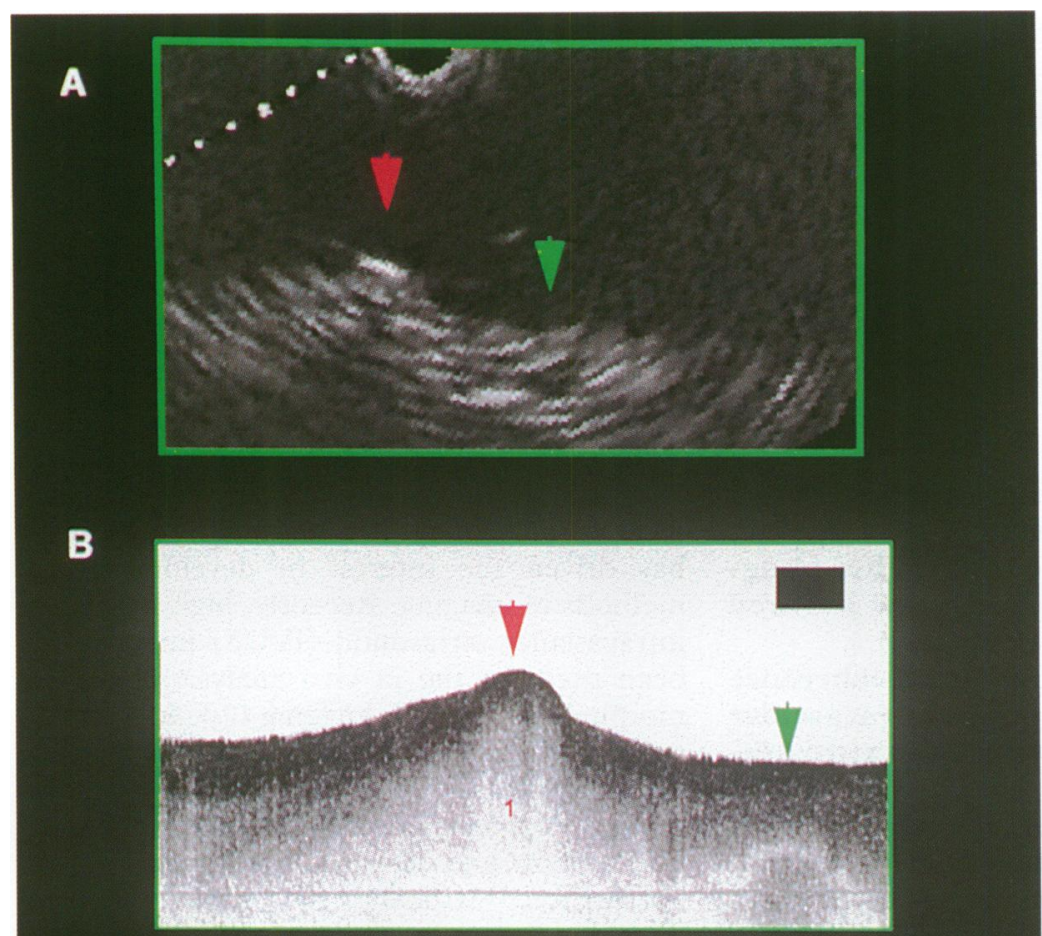

Reflectance

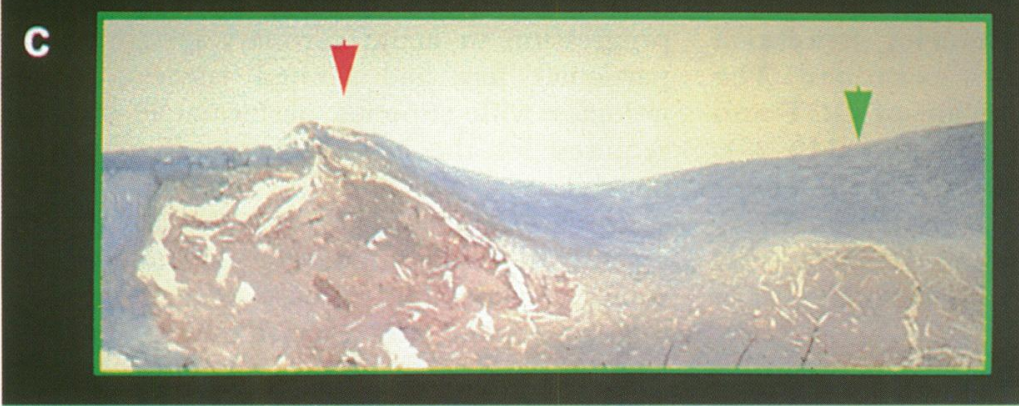

Figure 2 (A) IVUS and (B) OCT images, and (C) histogical specimen of a haemorrhagic plaque. $(A)$ The irregular luminal surface (red arrow) and a hypoechoic area are seen within the vessel wall (green arrow). White dots (upper left cormer) are $500 \mu \mathrm{m}$ apart. (B) OCT image of the plaque wall shows significantly greater structural detail than the ultrasound image. The plaque and hypoechoic area are differentiated, however, details that are not apparent with ultrasound imaging are evident with OCT. Bar represents $500 \mu \mathrm{m}$. (C) Histological specimen stained with trichrome blue. By comparison with the histology sample, it can be seen that the area of low optical back scattering within the centre of the plaque (red arrow) on the OCT image contains large quantities of lipid, matrix, and red blood cells with little cellular structures (1). This lack of cellular structure likely accounts for the low back reflecting signal from within the plaque. Both sides of the image appear to be layered near the surface. From the histological specimen, this represents relatively normal intima abutting a region with a high concentration of lipid and connective tissue. The ringed structure on the OCT image (green arrow) and the hypoechoic area on the IVUS image (green arrow) represent an area with a high content of lipid and matrix. The ringed appearance in the OCT image results from a continuous line of lipid crescents.

sue of the eye. ${ }^{113}$ Preliminary clinical studies in ophthalmology have shown that OCT can non-invasively image the retina with unprecedented resolution and should be a powerful diagnostic tool for a range of macular diseases. ${ }^{11} 1415$

We have recently developed new OCT techniques to image non-transparent tissue, notably aortic and coronary atherosclerotic plaques. ${ }^{1617}$ This has been achieved, in part, by performing imaging with longer wavelength light in the near infrared region to enhance imaging penetration depth. This article describes the first direct comparison between OCT and IVUS imaging, and assesses the relative performance of both modalities to image in vitro atherosclerotic aortic plaque morphology.

\section{Methods}

Aortas were obtained within six hours of postmortem examination. The samples were stored in physiological saline with $0.1 \%$ sodium azide at $0^{\circ} \mathrm{C}$. Imaging was performed with OCT and IVUS on segments smaller than $10 \times 10 \mathrm{~cm}$ with the luminal surface exposed. All IVUS samples and most OCT samples were immersed in saline (OCT does not require saline immersion and imaging can be performed through air). Twenty consecutive sites from five patients were examined by OCT followed by ultrasonic imaging under the direction of a separate, blind, experienced operator. No attempt was made to select for specific plaque morphology due to the preliminary nature of this work. The position of the OCT beam on the sample was monitored with a visible light guiding beam. The peripheral areas of imaged sections were marked with microinjections of dye. The position of the ultrasound beam on the tissue was confirmed through landmarks such as injected dye, abrupt changes in the luminal surface, and metal markers placed in the tissue for orientation. Imaging was performed with specimens at room temperature.

After imaging, the specimens underwent routine histological processing, being fixed in formalin for 24 hours and subsequently decalcified in a standard $\mathrm{Cal}$ Ex solution (Fisher Inc, St Louis, Missouri, USA) for 10 hours. The arteries were processed with routine paraffin embedding. Sections of $5 \mu \mathrm{m}$ thickness were cut at marked imaging sites, then stained with haematoxylin and eosin, and trichrome blue to identify different components of the vascular wall. Stained histological sections were compared with IVUS and OCT images to provide a better qualitative understanding of the reflectance properties of these tissue structures.

The principles behind OCT imaging have been previously described. ${ }^{11}{ }^{18-20}$ OCT functions analogously to ultrasound imaging except that it uses light rather than sound waves. Ultrasound imaging is performed by measuring the delay time (echo delay time) for an incident ultrasonic pulse to be reflected back from different internal structures within the tissue. Because the velocity of sound is relatively slow, this delay time may be measured electronically. OCT performs imaging by measuring the echo delay time of light reflected from internal tissue structures. In contrast to sound, the velocity of light is extremely high, and thus direct measurement of optical echoes cannot be performed electronically. ${ }^{21-23}$ OCT measures the echo delay time of light by interferometry.

Figure 1 represents a schematic diagram of the OCT device. OCT imaging is performed using "low coherence" light generated by a compact, infrared diode source which is cou- 


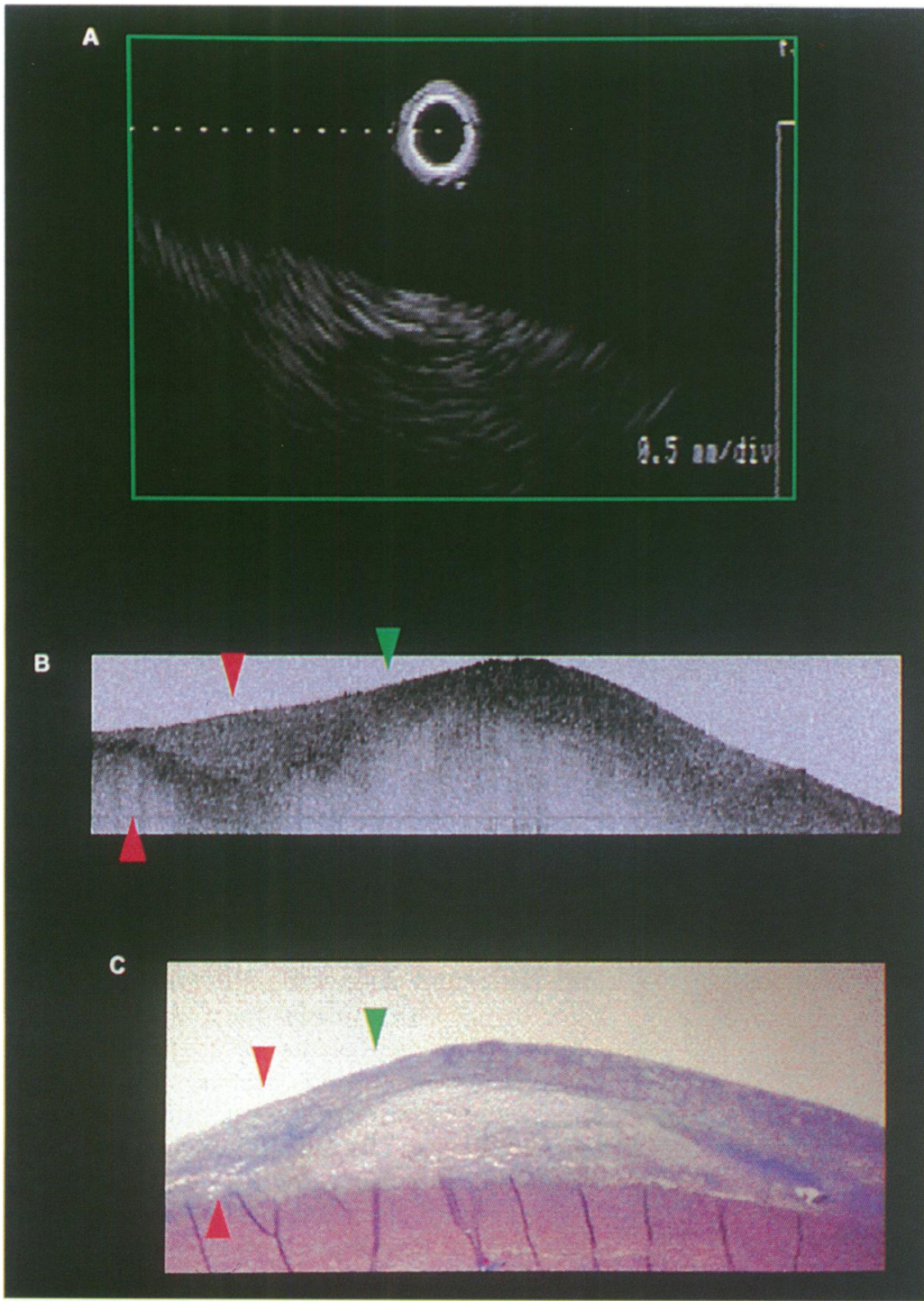

Figure 3 (A) IVUS and (B) OCT images, and (C) histogical specimen showing a large lipid laden plaque. OCT provides sharper delineation between the intimal wall and plaque. Good correspondence between thickness exists, with the green arrow demonstrating the thinnest area. Localised collections of lipid are identified with OCT but are not noted with IVUS imaging (red arrow). The elastic layer is poorly defined with both technologies. Therefore, the interface between the plaque and elastic layer is not well demarcated.

pled into an optical fibre. The light in the optical fibre is divided evenly by a fibre optical beam splitter into two fibres. One fibre is directed to the tissue sample and the other to a moving reference mirror. The position of the moving mirror is precisely controlled by the system electronics. Light is back reflected by the reference mirror and structures within the tissue. The back reflected beams are recombined by the optical beam splitter. Optical interference between the light from the sample and reference mirror occurs only when the optical distance travelled by the light in the tissue sample and reference paths matches to within the coherence length of the light. To visualise how low coherence interferometery works, low coherence light can also be thought of as a train of closely spaced pulses of light. The coherence length of the light is then analogous to pulse duration. Thus optical interference between the light pulse reflected from the tissue sample and the reference mirror can occur only when the reflected pulses coincide in time or alternately, when the path lengths that they travel are matched to within a pulse duration.

The use of low coherence interferometry permits the echo delay time (and the optical path length) of the light reflected from the tissue sample to be measured with extremely high accuracy. In addition, the magnitude of optical interference is a measurement of the intensity of optical back scatter or reflection from the tissue. When the reference mirror is scanned, the back scattered or reflected light from different depths within the tissue sample is measured. The result is measurement of optical back scatter or reflection versus axial range and is analogous to ultrasound $\mathrm{A}$ mode ranging.

The optical beam is scanned across the sample and sequential axial measurements are taken at different transverse positions to construct a two dimensional cross sectional image of the tissue specimen. The resultant two dimensional dataset represents the optical back scattering or reflection within a cross section of the tissue specimen. This dataset can be displayed as a two dimensional grey scale or false colour image. The magnitude of the optical back scatter is represented as a grey or colour scale to enhance differentiation of the tissue structures.

A superluminescent diode with a $1300 \mathrm{~nm}$ wavelength and a $50 \mathrm{~nm}$ band width (spectral width) was used as the light source for OCT imaging in this study. The spectral band width (axial resolution is inversely proportional to the spectral band width) yielded an axial spatial resolution of 16 (1) $\mu \mathrm{m}$. The resolution was verified by measuring the point spread function using a mirror. The lateral or transverse resolution of $30 \mu \mathrm{m}$ was measured with a Standard Air Force Resolution Chart. Unlike conventional microscopy, the axial resolution of OCT is determined by the coherence length of the light source and not the focusing parameters of the optical beam. Thus higher axial resolutions can be achieved by a shorter coherence length (broader bandwidth) light source.

The signal to noise ratio (SNR) was $109 \mathrm{~dB}$, using an intensity of $160 \mu \mathrm{W}$ at the sample. The SNR was determined by measuring the maximum detected signal when the optical beam is reflected from a mirror divided by the variance of the background noise level of the instrument. Images of backscattering intensity versus distance were displayed in grey scale or false colour. The axial dimension of the images correspond to $10 \mu \mathrm{m} / \mathrm{pixel}$. The acquisition times ranged from 20 to $45 \mathrm{sec}-$ onds, depending upon the size of the image acquired.

Ultrasonic imaging was performed with a $30 \mathrm{MHz}, 2.9$ French Microview ultrasonic transducer (Cardiovascular Instrument Systems, Sunnyvale, California), which has a mirror speed of $18000 \mathrm{rpm}$. The dynamic range was $43 \mathrm{~dB}$ (Cardiovascular Instrument Systems, personal communication, 1995) and is similar to other IVUS transducers. ${ }^{1024}$ Information was processed and displayed with an Insight III ultrasound system (Cardiovascular Instrument Systems). The image was 


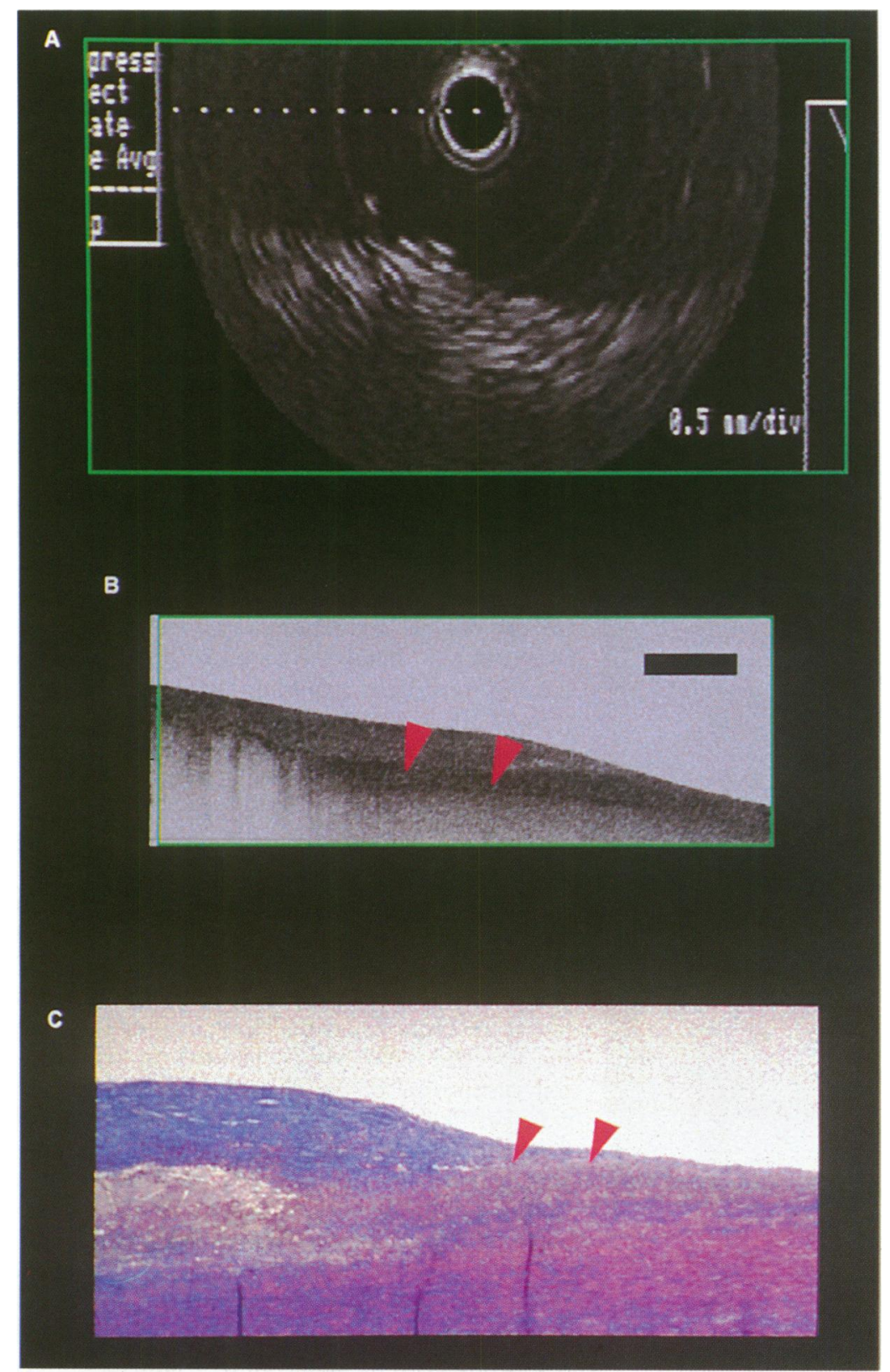

Figure 4 (A) IVUS and (B) OCT images, and (C) histogical specimen showing a large plaque. Superior structural information demonstrating layers within the adjacent plaque wall (arrows) is provided by OCT. Bar represents $500 \mu \mathrm{m}$.

oversampled at $18 \mu \mathrm{m} /$ pixel. Digital data from OCT and IVUS imaging were converted to analogue with IPLab (Signal Analytics, Vienna, Virginia) on a Power Macintosh 7100/66.

The point spread function was used to compare quantitatively the resolution of the two imaging technologies. ${ }^{25} 26$ Sound and light are strongly reflected at the surface of an interface such as that between air and metal. Thus the measured axial width of the returning signal is a measurement of the resolving power of the instrument. An imaging technology with an infinitely high resolution would resolve the surface as an infinitely sharp line. As any instrument has a finite resolution, however, the surface interface seems to have a finite width in the image. This is the point spread function and defines the axial resolution (the boundary cannot be resolved below the level of resolution of the instrument). The point spread functions for IVUS imaging and OCT were measured using a mirror. Digital data were converted to one dimensional data with image processing software (IPLab). Sampling was performed in the centre of the image and $1.5 \mathrm{~mm}$ in the transverse (lateral) direction to confirm that the resolution did not deteriorate because of the circular imaging field of the IVUS catheter. Widths were calculated at the full width half maximum. Values represent means (SE) of seven measurements.

\section{Results}

Figure 2 compares an OCT image of a haemorrhagic plaque with IVUS imaging and histology. In figure $2 \mathrm{~A}$, the IVUS image shows an irregularity in the luminal surface (red arrow) In addition, a hypoechoic area is seen within the vessel wall (green arrow). In figure $2 \mathrm{~B}$, the OCT image of the plaque wall shows significantly greater structural detail than the ultrasound image. The plaque and hypoechoic area are shown by OCT. Details that were not apparent with ultrasound assessment, however, become evident with OCT. By comparison with histology examination (fig 2C), the area of low back scattering within the plaque (1) contains large quantities of lipid, matrix, and red blood cells with little cellular structure. In the OCT image, the ringed structure (green arrow) represents an area with a high content of lipid and matrix. The ringed appearance resulted from a continuous line of lipid crescents. The left and right sides of the image appear to be layered where the relatively normal intima abuts a region with a high concentration of lipid and connective tissue.

Figure 3 shows OCT and IVUS images of a large lipid laden plaque. The lesion is also detected by ultrasound assesssment (arrow). OCT, however, provides a sharper delineation between intimal wall and plaque. Furthermore, localised collections of lipid are identified with OCT but are not observed with IVUS. The elastic layer is poorly defined with both imaging technologies. Previous work ${ }^{16}$ has shown that when lipid laden plaque abuts the elastic layer, the optical back scattering contrast between the two layers is relatively small at this wavelength $(1300 \mathrm{~nm})$. The use of alternate wavelengths for OCT imaging may lead to improved contrast between layers. Figure 4 shows a large plaque where layers in the adjacent wall are well demarcated. In figure 5, an intercostal artery emanating from the aorta is imaged with OCT and IVUS. Both imaging modalities demonstrate the presence of the vessel. However, structural detail is superior with OCT, illustrating the interweaving layers of tissue not differentiated by haematoxylin and eosin staining or IVUS but which are seen with trichrome blue staining (fig 5C).

Figure 6 shows the point spread function in the axial direction (which defines the axial resolution). The axial point spread function was $16(1) \mu \mathrm{m}$ for OCT and 110 (7) $\mu \mathrm{m}$ for IVUS at the centre of the image. Some $1.5 \mathrm{~cm}$ in the lateral (transverse) direction the resolutions 


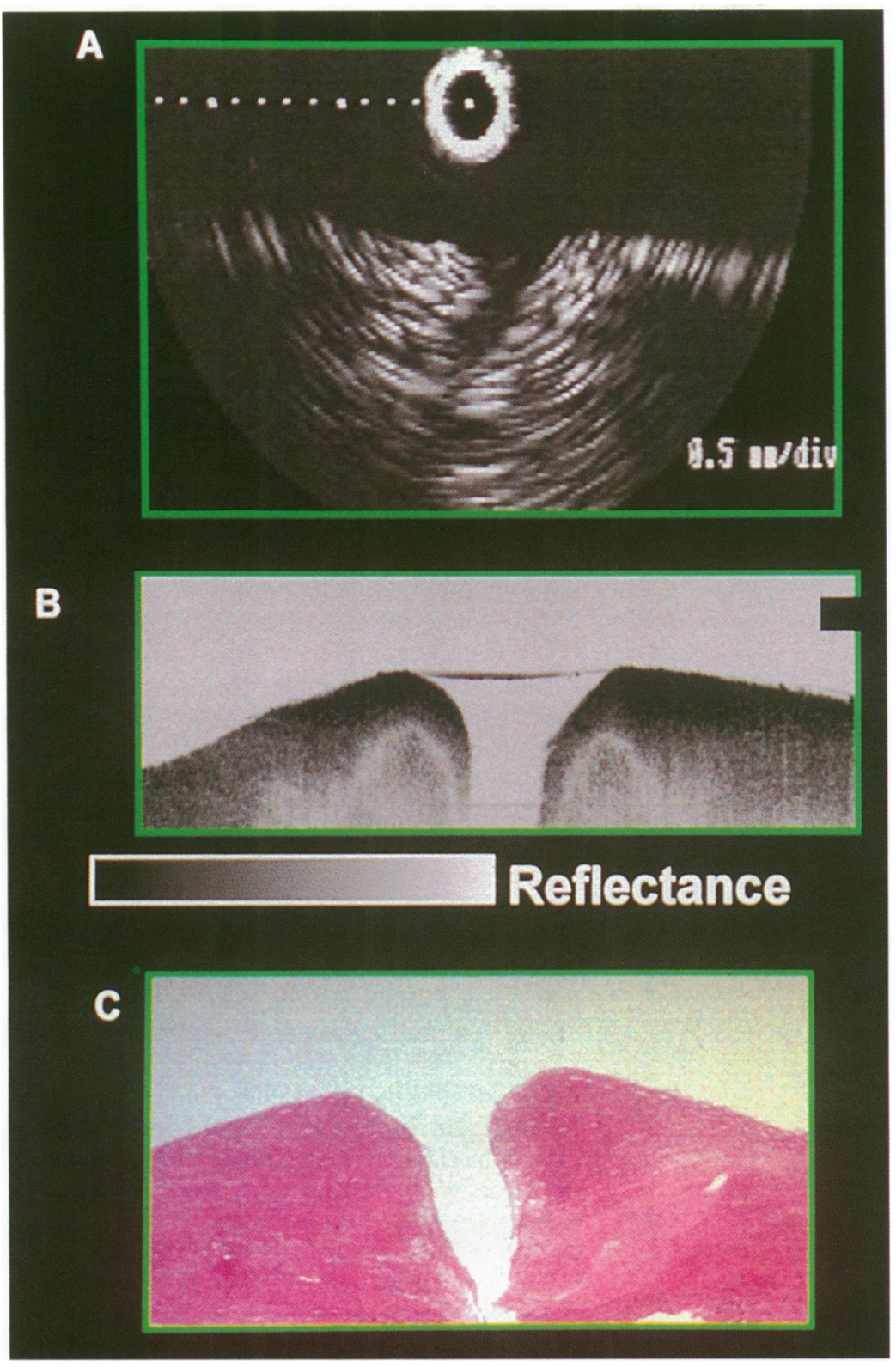

Figure 5 (A) IVUS and (B) OCT images, and (C) histogical specimen showing an intercostal artery emanating from the aorta. Structural detail is superior with OCT, illustrating the interweaving layers of tissue which are not evident with IVUS imaging. Bar represents $500 \mu \mathrm{m}$ identification of plaque microstructure should significantly improve patient risk stratification. In addition, an intravascular imaging technology with high resolution should improve efficiency and reduce complications associated with invasive catheter based treatment. Interventional procedures to manipulate atherosclerotic plaques could be used microsurgically if the guiding imaging technology could be extended to resolutions on a micrometre scale.

We have recently demonstrated the feasibility of OCT, a new method of micrometre scale imaging, for intravascular diagnostic investigation. ${ }^{1617}$ Earlier imaging studies ${ }^{16}$ of in vitro human aortic and coronary atherosclerotic plaques have shown that fissures, lipid collections, and intimal wall caps can be identified with unprecedented resolution. In the present study, OCT and IVUS were directly compared in terms of their ability to image in vitro aortic plaques.

The most striking advantage of OCT compared with that of ultrasound assessment is the consistently higher resolution and contrast between components of the plaques. The dynamic range and sensitivity of OCT $(-109 \mathrm{~dB}$ using an incident power of $160 \mu \mathrm{W})$ approaches the quantum limit, the theoretical maximum that can be achieved using optical detection. Although light is highly scattered in tissue, this high sensitivity and dynamic range permit imaging of tissue morphology to depths of 1-2 mm, a significant fraction of the vessel wall thickness. In contrast to ultrasound assessment, the contrast mechanism in OCT images arises from differences in the optical scattering properties of tissue rather than acoustic scattering properties. This leads to the dramatic difference in contrast between adjacent areas of distinct tissue composition, which is most prominently seen in figures 2-4. The high resolution, which was evident qualitatively in the images, was confirmed by measuring the point spread function at an interface with both imaging modalities. The $16 \mu \mathrm{m}$ resolution of OCT was five times greater than the $110 \mu \mathrm{m}$ measured for IVUS. Furthermore, the resolution of OCT can be increased to the $4 \mu \mathrm{m}$ range with the use of broad band width or femtosecond sources. ${ }^{12}$ Finally, multiple echoes that can lead to image distortion were observed with IVUS. ${ }^{27}$ Multiple echoes result because sound waves, reflected off the tissues, are also reflected off the surface of the ultrasound transducer (a high acoustical mismatch exists between the transducer and saline/blood). Echo artefacts were not observed with OCT.

The technology on which OCT is based is well suited for clinical use. Because OCT uses optical fibre technology and compact diode laser light sources, it can be engineered into a clinically compatible system. The OCT unit is compact and portable, similar in size to a standard external defibrillator or personal computer, making it attractive for clinical use. OCT is based on fibre optics that are used in optical communications technology, so that an optical fibre (with a standard diameter of technologies superior to those currently available will likely be required to identify these plaque features in vivo as the best current imaging technology, IVUS, does not provide sufficient resolution and contrast. ${ }^{689}$ Improved 

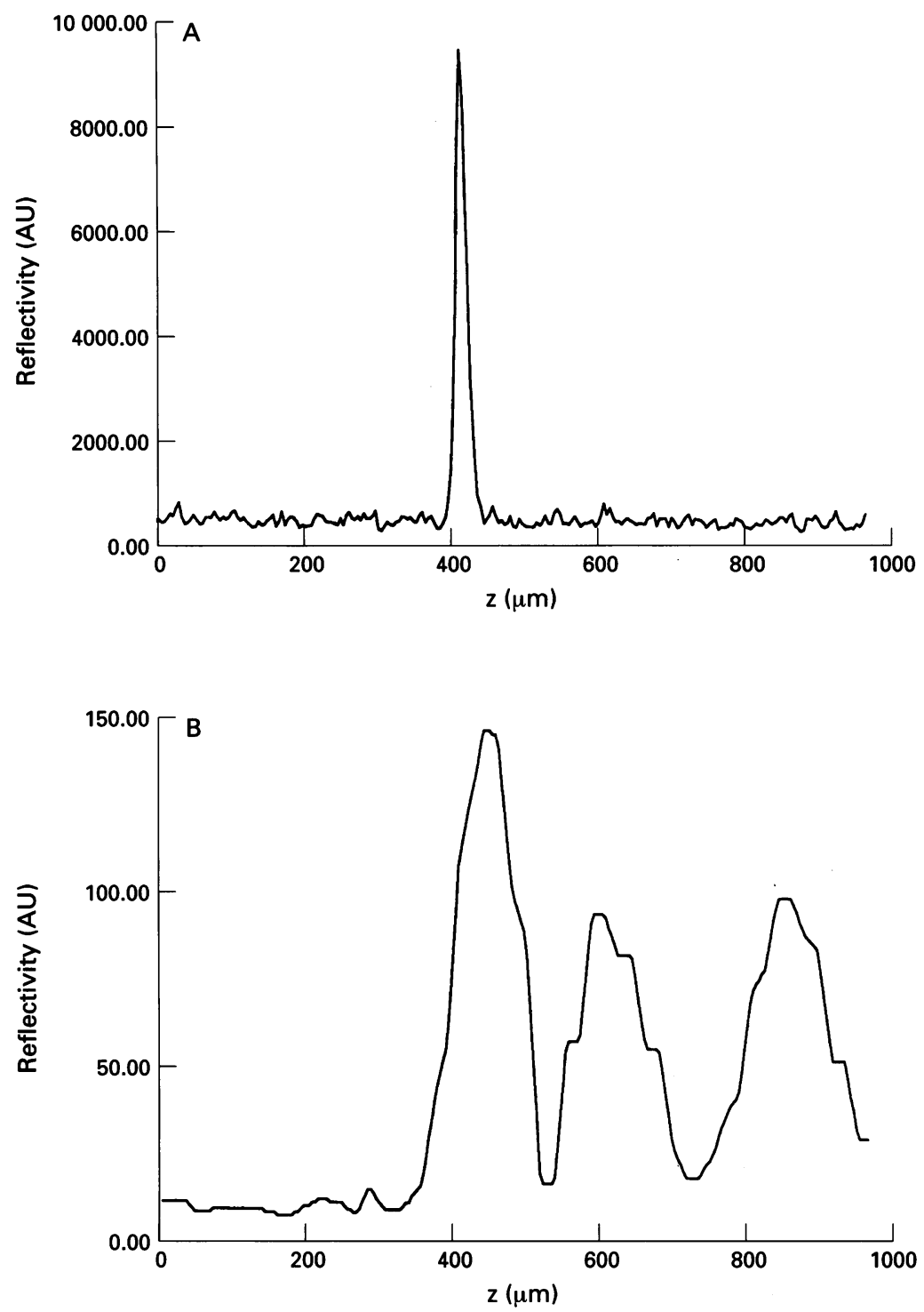

Figure 6 Representative point spread functions, defining axial resolution, are shown for (A) OCT and (B) IVUS imaging. The axial point spread functions were 16 (1) $\mu \mathrm{m}$ for OCT and 110 (7) $\mu \mathrm{m}$ for IVUS at the centre of the image, while $1.5 \mathrm{~cm}$ lateral the measured resolutions were 15 (1) $\mu \mathrm{m}$ and 107 (7) $\mu \mathrm{m}$, respectively. Multiple echoes are seen with IVUS imaging but not with OCT.

$125 \mu \mathrm{m})$ can be readily integrated into an intravascular catheter. Implementation will be similar to that of the ultrasound catheter, with a reflecting and focusing element used on the distal tip. However, no transducer is required in the catheter. The ultimate catheter should resemble the IVUS catheter in size. Transit time or ring down restrictions, which limit the rate of data acquisition for ultrasound, are not present with OCT as the source and detector are separate elements. ${ }^{34}$ Finally, as the absorption of light, unlike sound, is strongly dependent on molecular composition, OCT has the potential for performing in vivo biochemical analysis based on spectroscopic properties.

A number of practical issues must be addressed before clinical trails may be implemented. Future investigation will include methods for reducing image acquisition time, determining the imaging properties through blood, increasing imaging penetration, and the development of OCT and catheter technology for future in vivo imaging. The acquisition times used in this study are inadequate for in vivo imaging, but imaging time may be reduced significantly. Instrument improvements including increasing source power, scanning speed, and redesigning of electronics are straightforward and should lead to substantial reductions in imaging time. The imaging properties of blood are unknown. Although blood strongly absorbs visible light, it has very low absorption in the infrared region. Optical scattering, however, may reduce the amount of optical signal reflected from the vessel wall. The imaging properties of blood will be the topic of future investigation. If blood leads to a significant reduction in image quality, simultaneous injections of saline may be required for in vivo use similar to that needed in angioscopy. Unlike IVUS, imaging with OCT can be performed in air or saline without appreciable loss of image quality.

The penetration depths for IVUS versus OCT were not directly compared in this study as the focus of our investigation was to compare resolution. In general, ultrasound assessment is capable of imaging significantly deeper than optical techniques because attenuation and scattering are generally less for acoustic waves at $30 \mathrm{MHz}$ than for light. The results presented in this study, however, suggest that OCT imaging depth will be sufficient for the diagnosis of a range of clinically significant coronary pathologies. OCT with a $1300 \mathrm{~nm}$ light source allows imaging through the width of a normal proximal coronary artery $(2 \mathrm{~mm}) .{ }^{12}$ In addition, we have previously demonstrated that changing the wavelength of the incident light from $850 \mathrm{~nm}$ to $1300 \mathrm{~nm}$ results in significant improvements in imaging penetration. ${ }^{16}$ Furthermore, optimisation of the wavelength and increasing the incident power (which is well below the level set by safety standards) will likely result in substantial improvements in OCT imaging penetration.

In conclusion, OCT imaging of plaque microstructure consistently demonstrates superior resolution and contrast when compared with that of IVUS. OCT represents a promising new technology for intravascular imaging due to its high resolution, broad dynamic range, and ability to be adapted for catheter based imaging.

We thank Ms Cindy Kopf for her assistance in the preparation of this manuscript, and Joe Gamba, Kathryn Blackwell, and this manuscript, and Joe Gamba, Kathryn Blackwell, and James Taralli for their technical support. This work was supported in part by National Institute of Health grants NIH-5HL55686-01A1, the Office of Naval Research Medical Free HL55686-01A1, the Office of Naval Research Medical Free
Electron Laser Program contract N00014-94-1-0717, and Electron Laser Program contract N0001
Whittaker Foundation contract 96-0205.

1 Nishimura RA, Edwards WD, Warnes CA, Reeder GS Holmes DR, Tajik AJ, et al. Intravascular ultrasound imaging: in vitro validation and pathologic correlation. Am Coll Cardiol 1990;16:145-54.

2 Gussenhoven EJ, Essed CE, Lancee CT, Mastik F, Frietman $\mathrm{P}$, Egmond FCV, et al. Arterial wall characteristics determined by intravascular ultrasound imaging: an in vitro study. $7 \mathrm{Am}$ Coll Cardiol 1989;14:947-52.

3 Yock PG, Fitzgerald PJ, Linker DT, Angelsen BA Intravascular ultrasound guidance for catheter-based coronary interventions. $\mathcal{f}$ Am Coll Cardiol 1991;17:39B45B.

4 Mudra H, Klauss V, Blasini R, Kroetz M, Rieber J, Regan E. Ultrasound guidance of Palmaz-Schatz intracoronary stenting with a combined intravascular ultrasound balloon catheter. Circulation 1994;90:1252-61. 
5 Nissen SE, Gurley JC, Booth DC, DeMaria AN. Intravascular ultrasound of the coronary arteries: current applications and future directions. Am $\mathcal{f}$ Cardiol 1992;69: $18 \mathrm{H}-29 \mathrm{H}$.

6 Lee DY, Eigler N, Luo H, Steffen W, Tabak S, Seigel RJ. Intravascular coronary ultrasound imaging, is

7 Lee DY, Eigler N, Luo H, Wishioka T, Tabak SW, Forrester JS. Effect of intracoronary ultrasound imaging on clinical decision making. Am Heart $\mathcal{f}$ 1995;129: 1084-93.

8 Peters RJ, Ge J, Linker DT, Visser CA, Yock PG. Observer agreement on qualitative analysis of intracoronary ultraagreement on qualitative analysis of intrac

9 Peters RJ, Kok WE, Havenith MG, Rijsterborgh H, van der Wal AC, Visser CA. Histopathologic validation of intracoronary ultrasound imaging. $₹ \mathrm{Am}$ Soc Echocardiogr coronary ultrasoun

10 Hibberd MG, Vuille C, Weyman AE. Intravascular ultrasound: basic principles and role in assessing arterial morphology and function. Am $f$ Card Imaging 1992;6: 308-24.

11 Huang D, Swanson EA, Lin CP, Schuman JS, Stinson WG, Chang W. Optical coherence tomography. Science $1991 ; 254: 1178-81$.

12 Bouma BE, Tearney GJ, Boppart SA, Hee MR, Brezinski $M E$, Fujimoto JG. High resolution optical coherence tomographic imaging using a modelocked $\mathrm{Ti}: \mathrm{Al}_{2} \mathrm{O}_{3}$ laser source. Opt Lett 1995;20:1486-8.

13 Swanson EA, Izatt JA, Hee MR, Huang D, Lin CP, Schuman JS. In vivo retinal imaging by optical coherence tomography. Opt Lett 1993;18:1864-6.

14 Hee MR, Izatt JA, Swanson EA, Huang D, Lin CP, Schuman JS. Optical coherence tomography of the human retina. Arch Ophthalmol 1995;113:325-32.

15 Puliafito CA, Hee MR, Lin CP, Reichel E, Schuman JS, Duker JF. Imaging of macular diseases with optical coherence tomography. Ophthalmology 1995;102:217-29.

16 Brezinski ME, Tearney GJ, Bouma BE, Izatt JA, Hee MR Swanson EA. Optical coherence tomography for optical biopsy: properties and demonstration of vascular patholbiopsy: properties and demonstration

17 Brezinski ME, Tearney GJ, Bouma BE, Boppart SA, Hee MR, Swanson EA. Imaging of coronary artery microMR, Swanson EA. Imaging of coronary artery microstructure with optical
Cardiol 1996;77:92-3.

18 Takada K, Yokohama I, Chida K, Noda J. New measurement system for fault location in optical waveguide devices based on an interferometric technique. Appl $O p t$ 1987;26:1603-6.
19 Huang D, Wang J, Lin CP, Puliafito CA, Fujimoto JG. Micron-resolution ranging of cornea anterior chamber by optical reflectometry. Laser Surg Med 1991;11:419-25.

20 Fercher AF, Mengedoht K, Werner W. Eye-length measurement by interferometry with partially coherent light. Opt Lett 1988;13:186-90.

21 Clivaz X, Marquis-Weible F, Salathe RP, Novak RP, Gilgen $\mathrm{HH}$. High-resolution reflectometry in biological tissues. Opt Lett 1992;17:4-6.

22 Schmitt JM, Knuttel A, Bonner RF. Measurements of optical properties of biological tissues by low-coherence reflectometry. Appl Opt 1993;32:6032-42.

23 de Wolf DA. Coherence of a light beam through an optically dense turbid layer. Appl Opt 1978;17:1280-5.

24 Fitzgerald PI, St Goor FG, Connolly AJ, Pinto FJ, Billington ME, Popp RL. Intravascular ultrasound imaging of coronary arteries: is three layers the norm? ing of coronary arteries:

25 Haus HA. Waves and fields in optoelectronics. Englewood Cliffs, New Jersey: Prentice Hall, 1984:5-35.

26 Swanson EA Huang D, Hee MR, Fujimoto JG, Lin CP, Swanson EA, Huang D, Hee MR, Fujimoto JG, Lin CP,
Puliafito CA. High-speed optical coherence domain Puliafito CA. High-speed optical

27 Finet G, Maurincomme E, Tabib A, Crawley RJ, Magnin I, Roriz R. Artifacts in intravascular ultrasound imaging: analyses and implications. Ultrasound Med Biol 1993; 19:533-47.

28 Herrick JB. Clinical features of sudden obstruction of the coronary arteries. $\mathcal{F} A M A$ 1912;59:2015-20.

29 Richardson PD, Davies MJ, Born GVR. Influence of plaque configuration and stress distribution on fissuring of coronary atherosclerotic plaques. Lancet 1989;i:941-4.

30 Falk E. Plaque rupture with severe pre-existing stenosis precipitating coronary thrombosis, characteristics of coronary atherosclerotic plaques underlying fatal occlusive thrombi. Br Heart $\mathcal{f}$ 1983;50:127-34.

31 Davies MJ, Thomas AC Plaque fissuring - the cause of acute myocardial infarction, sudden ischemic death, and crescendo angina. Br Heart $\mathcal{f} 1985 ; 53: 363-73$.

32 Fuster V, Badimon L, Badimon JJ, Chesebro JH. The pathogenesis of coronary artery disease and the acute coronary syndromes. N Engl ₹ Med 1992;326:242-50.

33 Ambrose JA, Tannenbaum MA, Alexopoulos D, Hjemdahl-Monsen CE, Leavy J, Weiss M. Angiographic progression of coronary artery disease and the developprogression of coronary artery disease and the development of

34 Benkeser BJ, Churchnell AL, Lee C, Abouelnosp DM. Resolution limitations of intravascular imaging. $7 \mathrm{Am} \mathrm{Soc}$ Echo 1993;6:158-65. 